\title{
Superconducting proximity effect in a mesoscopic ferromagnetic wire
}

\author{
M. Giroud ${ }^{1}$, H. Courtois ${ }^{1}$, K. Hasselbach ${ }^{1}$, D. Mailly ${ }^{2}$, and B. Pannetier ${ }^{1}$ \\ ${ }^{1}$ Centre de Recherches sur les Très Basses Températures-C.N.R.S. associated to Université Joseph Fourier, 25 Ave. des \\ Martyrs, 38042 Grenoble, France \\ ${ }^{2}$ Laboratoire de Microstructures et de Microélectronique-C.N.R.S., 196 Ave. H. Ravera, 92220 Bagneux, France
}

(October 24, 2018)

We present an experimental study of the transport properties of a ferromagnetic metallic wire $(\mathrm{Co})$ in metallic contact with a superconductor $(\mathrm{Al})$. As the temperature is decreased below the $\mathrm{Al}$ superconducting transition, the Co resistance exhibits a significant dependence on both temperature and voltage. The differential resistance data show that the decay length for the proximity effect is much larger than we would simply expect from the exchange field of the ferromagnet.

74.50.+r, 74.80.Fp, 85.30St

Superconducting proximity effect consists in inducing superconductive properties in a non-superconducting meta. Although this effect has been studied for a long time1, it has gained some renewed interest due to recent experiments performed on samples of mesoscopic size. In such samples, the electron phase-breaking length $L_{\varphi}$ is larger than the sample length $L$. One can thus probe experimentally the characteristic energy scale of the proximity effect $\epsilon_{c}=\hbar D / L^{2}$, which is the Thouless energy related to the sample length. This has led for instance to the observation of large magnetoresistance oscillations in normal metal (A) loops in contact with a superconducting $(\mathrm{S})$ island $\mathrm{E}$. These oscillations provide a direct evidence for the long-range (up to $L_{\varphi}$ ) nature of the proximity effect. Another recent and striking result is the reentrant behaviour. The excess conductance induced by proximity effect is maximum at a temperature or a bias voltage equivalent to the sample Thouless energyb, but the normal state conductance reappears at lower energy.

Most experiments were performed in noble metals or semiconductor 2D electron gas, where electron interactions are negligible. In a free electron model, the zerotemperature, zero-bias resistance of a mesoscopic metar

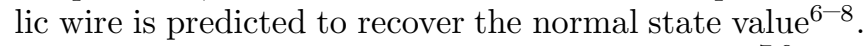
In the presence of interactions, theoretical studies 0 . predict a severe modification of the transport properties. Attractive (respectively repulsive) electron-electron interactions are believed to result in a resistance lower (respectively higher) than the normal-state oned. This could provide a probe for interactions in normal metals like $\mathrm{Au}$, $\mathrm{Ag}$, etd 10 .

In this communication, we present an experimental study of the superconducting proximity effect in a ferromagnetic metal $(\mathrm{F})$. Magnetic metals are in the strong interaction limit. Exchange interactions between electrons in a ferromagnet usually lead to efficient Cooper- pair breaking in F-S structures. However, it is worthwhile re-examining the actual proximity eff ferromagnetic wire 11 . Some experiments 12,13 suggested long-range coherence effects, but without any clear conclusion.

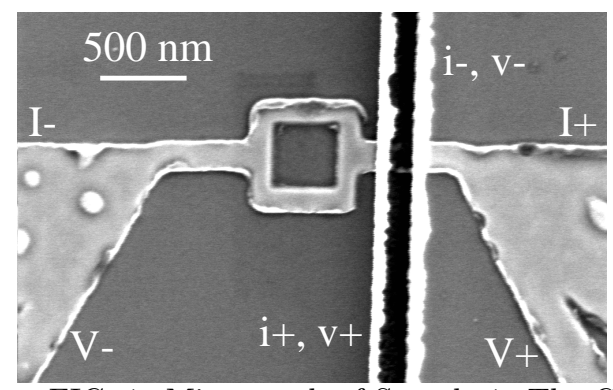

FIG. 1. Micrograph of Sample 1. The Co wire and loop is in metallic contact with one $\mathrm{Al}$ island. Thin residual Al strips appear on the sides of the $\mathrm{Al}$ wire. The side length of the Co loop is $500 \mathrm{~nm}$. The four Co contact pads are labelled I+, I-, $\mathrm{V}+, \mathrm{V}-$. The $\mathrm{Al}$ island is patterned with four contacts $\mathrm{i}+$, i-, $\mathrm{v},+\mathrm{v}-$, as indicated. In sample 2 (not illustrated), a second $\mathrm{Al}$ island is patterned on the left-hand side on the Co loop.

Samples (see Fig. 1) were fabricated using a two-step lift-off process. The $50 \mathrm{~nm}$ thick Co layer was e-beam evaporated on the patterned resist that was subsequently lifted off. The $100 \mathrm{~nm}$ thick $\mathrm{Al}$ islands were deposited after a soft in-situ ion-milling of the Co surface. The insitu cleaning is a crucial step to achieve the desired high transparency of the $\mathrm{Co}-\mathrm{Al}$ interface. In order to generate interferences, the Co conductor included a $0.5 \mu \mathrm{m}$ square loop. The distance between the Co reservoirs is $2 \mu \mathrm{m}$. Many samples were patterned on the same substrate, with zero, one or two $\mathrm{Al}$ islands. In the last two cases, one $\mathrm{Al}$ island was also linked to four contacts, in order to measure the $\mathrm{Al}$ wire and the $\mathrm{Co} / \mathrm{Al}$ junction resistances. The width of the $\mathrm{Co}$ and $\mathrm{Al}$ wires were 100 and $140 \mathrm{~nm}$ respectively. Here we will focus on two typical samples labelled 1 and 2, with one and two $\mathrm{Al}$ islands respectively. The behaviour of each of these two samples is representative of the properties of four samples we measured.

Figure 2 shows the temperature dependence of the resistance of samples 1 and 2 . The normal-state resistance of samples 1 and 2 is $96.09 \Omega$ and $98.35 \Omega$ respectively. With a Fermi velocity of $1.9 .10^{6} \mathrm{~m} / \mathrm{s}$ in Co, we get an elastic mean free path $l_{e}$ of $1.1 \mathrm{~nm}$ and a diffusion coefficient $D=v_{F} l_{e} / 3$ of $6.9 \mathrm{~cm}^{2} / \mathrm{s}$. As the temperature is decreased below the $\mathrm{Al}$ superconducting transition, the 
resistance of both samples decreases, reaches a minimum around $0.9 \mathrm{~K}$ and then increases. The temperature for the resistance minima is slightly different in the two samples, we do not have a simple explanation for that. The total amplitude of the variations is about $0.3 \%$ in sample 1 and $0.8 \%$ in sample 2 . In both cases, the lowtemperature saturation value of the resistance is larger than the normal- state resistance.

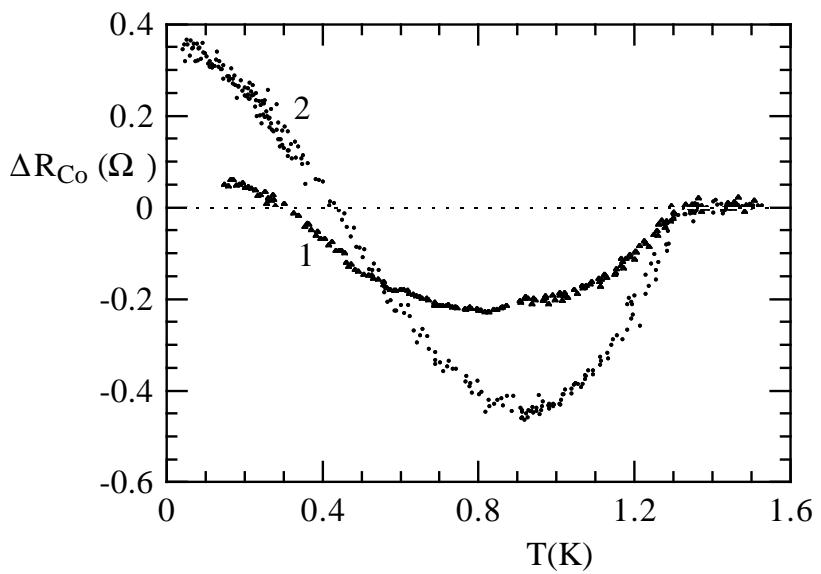

FIG. 2. Temperature dependence of the resistance of sample 1 and 2. Sample 1 has one Al island in contact with the Co loop, sample 2 has two. The normal-state resistance, respectively $96.09 \Omega$ and $98.35 \Omega$, has been subtracted. Bias current $0.1 \mu A$.

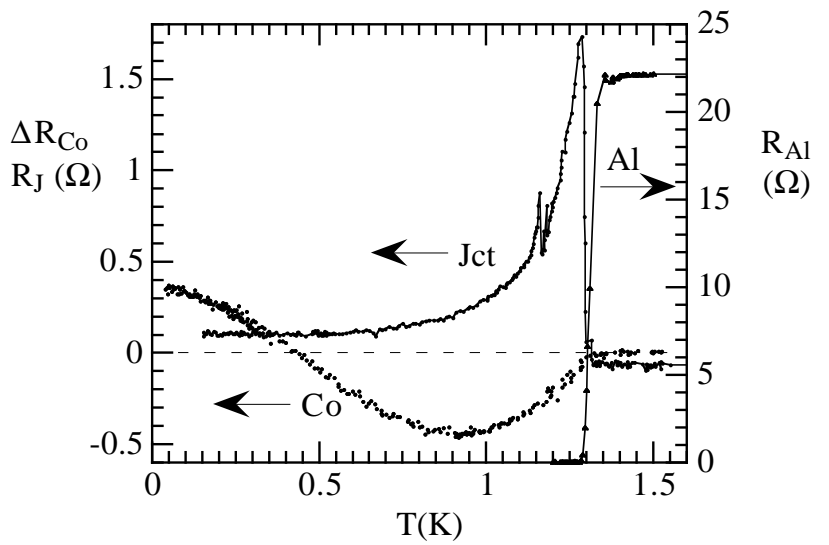

FIG. 3. Temperature dependence of the resistance of the $\mathrm{Al}$ wire (right hand scale), the Co wire and the $\mathrm{Co} / \mathrm{Al}$ junction (left hand scale) of sample 2. Bias current $0.1 \mu \mathrm{A}$.

Figure 3 shows the same data for sample 2, together with the resistance of its $\mathrm{Al}$ wire and of the $\mathrm{Co} / \mathrm{Al}$ junction. The Al wire becomes superconducting at $1.34 \mathrm{~K}$, with a transition width of about $10 \mathrm{mK}$. The superconducting properties of the $\mathrm{Al}$ wire are not strongly depressed by the proximity of the Co interface.

We measured the junction resistance by injecting current from one side $(\mathrm{I}+)$ of the Co wire to one side of the Al wire (i-) and measuring the voltage drop between the opposite sides of the $\mathrm{Co}$ and $\mathrm{Al}$ wires $(\mathrm{V}-$ and $\mathrm{v}+$ ). The small negative offset above $1.34 \mathrm{~K}$, when the $\mathrm{Al}$ wire is normal, stems from a 3-dimensional spreading of the current lines in the metallic electrodes of the junction. Such a sign reversal in a crossed-shaped junction only occurs when the resistance of the junction is significantly smaller than the electrodes resistances (here, $10 \Omega$ and $0.4 \Omega$ respectively for $\mathrm{Co}$ and $\mathrm{Al}$ ). This argument together with the measured resistance of $0.1 \Omega$ at the lowest temperature when the $\mathrm{Al}$ wire is superconducting confirms that our junction is metallic. This order of magnitude is consistent with a transparency $t$ of a few $\%$ after the relation $R_{t}^{-1}=2 N\left(E_{F}\right) v_{F} S e^{2} t$. We believe that the junction resistance peak below $1.34 \mathrm{~K}$ is related to charge-imbalance effects in the $\mathrm{Al}$ island, when the gap is small compared to the injected quasiparticle energy. The Co resistance change is not much larger than the Al junction one, but we stress that the Co resistance varies significantly below $0.5 \mathrm{~K}$, whereas the junction resistance does not vary anymore. This clearly shows that the variation of $\mathrm{Co}$ resistance is not due to a current redistributigneffect induced by variations of the junction resistance 14 .

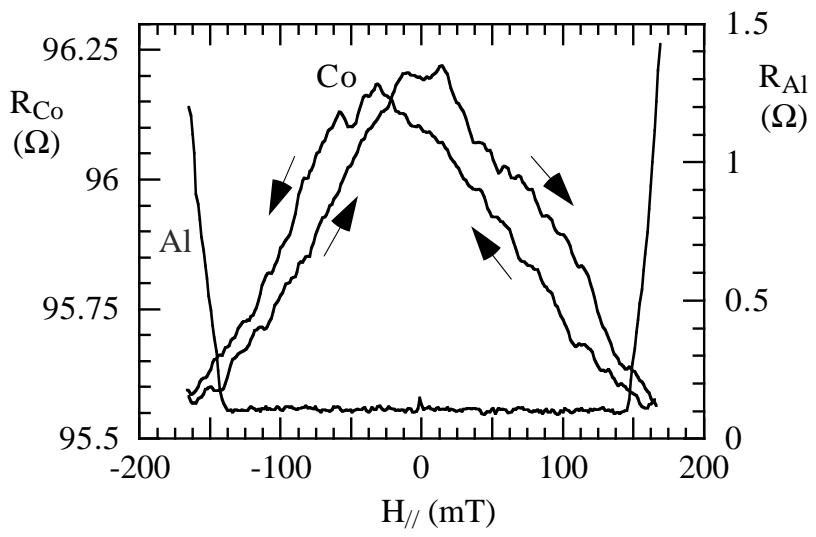

FIG. 4. Magnetoresistance of sample 1 Co wire and $\mathrm{Al}$ wire. The magnetic field is in the substrate plane, parallel to the Al wire. The Co resistance is hysteretic. The superconducting critical field of $\mathrm{Al}$ is close to $140 \mathrm{mT}$. Bias current $0.1 \mu A$.

The magnetoresistance of sample 1 and 2 was studied in magnetic fields applied perpendicular or parallel to the substrate. Figure 4 shows the magnetoresistance of sample 1 in parallel field. From the $\mathrm{Al}$ resistance, we measure a critical field of $140 \mathrm{mT}$ for the Al wire. The Co wire has a small (less than $1 \%$ at $140 \mathrm{mT}$ ) and hysteretic magnetoresistance. No saturation is visible up to 170 $\mathrm{mT}$. From this measurement, we can assert that our Co wire is ferromagnetic, but that all our experiments were performed in a regime where the Co magnetisation is not saturated.

Figure 5 shows the magnetoresistance of the Co wire in perpendicular field, above the superconducting transition of $\mathrm{Al}$ at $1.5 \mathrm{~K}$, and well below at $0.29 \mathrm{~K}$. There is no magnetoresistance at $1.5 \mathrm{~K}$, indicating that the $\mathrm{Co}$ magnetisation is in-plane. The perpendicular negative 
magnetoresistance only appears when $\mathrm{Al}$ becomes superconducting, and is about 8 times smaller than in parallel field. We searched for periodic oscillations of magnetoresistance as a function of perpendicular field. We achieved a resistance resolution better than $10^{-5}$, corresponding to $10^{-3} e^{2} / h$, by averaging over a large number of scans. The absence of Aharonov-Bohm oscillations at this level is a strong indication that the phase-breaking length in Co is smaller than $0.3 \mu \mathrm{m}$. This casts some doubts on the possible observatipn of weak-localisation-like effects in ferromagnetic films 13 .

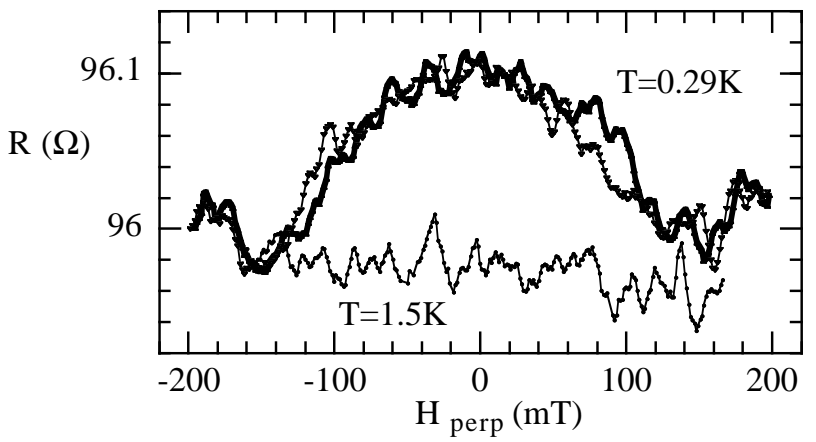

FIG. 5. Magnetoresistance of sample 1 at $\mathrm{T}=0.29 \mathrm{~K}$ and $\mathrm{T}=1.5 \mathrm{~K}$ in perpendicular field. Field scan from -200 to 200 , then back to $-200 \mathrm{mT}$. No periodic oscillations of the resistance were visible above the experimental noise. Bias current $0.1 \mu \mathrm{A}$.

We measured the differential resistance as a function of the bias current. Figure 6 shows three such curves recorded in different conditions. The upper curve was recorded at the lowest temperature $32 \mathrm{mK}$. The differential resistance exhibits a peak at zero bias, a minimum in the $1.7 \mu \mathrm{A}$ range, and returns to the normal state value at high bias. This is strongly reminiscent of the re-entrance effect5. We estimate that Joule heating did not exceed $0.3 \mathrm{~K}$ in the range below $5 \mu A$, and we checked that the junction resistance did not vary in this bias range.

The lower two curves of Fig. 6 have been shifted for clarity. The middle curve was recorded at a temperature of $0.8 \mathrm{~K}$. This curve looks very similar to the first one, with the remarkable exception that the zero-bias maximum is absent. In contrast, the high-bias features are unchanged. The lowest curve was recorded at very low temperature, in a parallel field of $130 \mathrm{mT}$, just below the Al critical field of $140 \mathrm{mT}$. This last curve shows a clear re-entrance peak of differential resistance below $1.0 \mu \mathrm{A}$, but the high-bias peaks are no longer present.

Let us compare these results with the ones previously reported for $\mathrm{Cu}$. On increasing current, and thus voltage, we increase the energy of the electrons injected in the F-S mesoscopic sample. We can thus probe the energy dependence of the proximity-induced excess conductance. As in a "free electron" metal, we observe a resistance minimum both as a function of temperature and bias current. The differential resistance is maximum at zero temperature and voltage : this is the re-entrance effect for the metallic conductance of the normal metal.

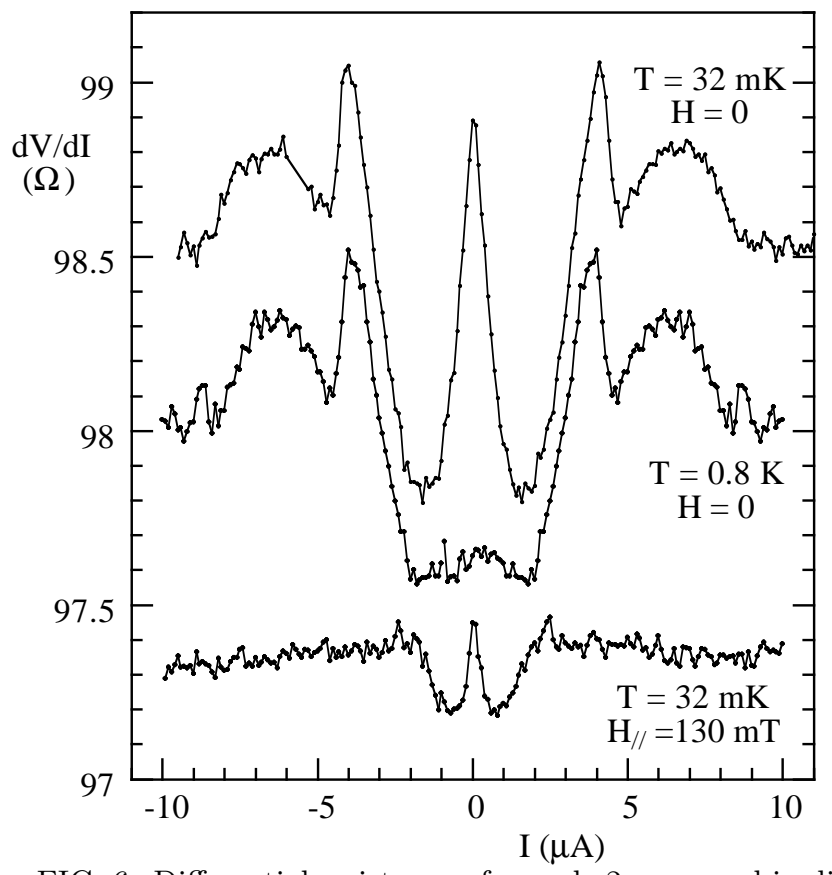

FIG. 6. Differential resistance of sample 2 measured in different conditions. Upper curve : $\mathrm{T}=32 \mathrm{mK}, \mathrm{H}=0$. Middle curve : $\mathrm{T}=0.8 \mathrm{~K}, \mathrm{H}=0$. Lower curve : $\mathrm{T}=32 \mathrm{mK}, \mathrm{H}$ $=130 \mathrm{mT}$ (parallel to the substrate). The latter two curves have been offset by 0.5 and $1 \Omega$ respectively for clarity.

The high-bias peaks are related to the Al superconducting gap and/or critical current. At $\mathrm{T}=32 \mathrm{mK}$, the $130 \mathrm{mT}$ magnetic field strongly depresses the Al gap, but does not affect the characteristic energies of the electrons injected in the F-S sample. Consequently, the reentrance peak of the differential resistance at zero energy is still visible, but the high bias peaks disappear. On the other hand, at $0.8 \mathrm{~K}$ and zero field, most of the electrons have an energy above the characteristic energy of the reentrance effect, but the $\mathrm{Al}$ gap is not yet depressed, so that only the zero bias maximum disappears. This is consistent with our picture of the proximity effect.

In the quasiclassical theory, the temperature of the resistance minimum is $5 \epsilon_{c} / k_{B}$. The temperature dependence data gives us a Thouless energy of $14 \mu \mathrm{eV}$ for the sample 2. With our estimate of the diffusion coefficient in Co, this energy would give a characteristic length of $180 \mathrm{~nm}$, much shorter than the total sample length of $2 \mu \mathrm{m}$. A simple interpretation of this result is that the Co electrons reflected on the $\mathrm{Al}$ island keep their phase coherence only on this shorter length scale. The effective mesoscopic sample length we are probing is only $180 \mathrm{~nm}$. It is also the order of magnitude of magnetic demain sizes for Co samples deposited in similar conditions 15 . Indeed, it has been suggested that dgmain walls could contribute to decoherence of electrons 16 .

This decay length is much larger than the "exchange length" : $L_{\text {exch }}=\sqrt{\hbar D / k_{B} T_{\text {Curie }}}=2 \mathrm{~nm}$ in the dirty 
limit $\left(L_{\text {exch }}>l_{p}\right)$, with $T_{\text {Curie }}=1388 \mathrm{~K}$ being the Curie temperature of Co. This length scale arises from the magnetic energy splitting between the incident electron and the Andreev reflected hole in the exchange field of $\mathrm{F}$. $\mathrm{D}$. In the Andreev reflection process, the electron spin is reversed. In consequence, the reflected hole has a different energy and momentum than the incident electron. This results in a finite decay length $L_{\text {exch }}$.

If we take sample 2 normal state resistance $(98.35 \Omega)$ to convert the current bias into a voltage corresponding to the minimum differential resistance, we get $170 \mu \mathrm{eV}$. This is about 2.4 times larger than the voltage derived from the temperature dependence (Fig. 2), and even larger than the $\mathrm{Al}$ gap. This confirms that the coherence effects only occur on a length scale shorter than the total wire length. In comparison, $180 \mathrm{~nm}$ of our Co wire would have a resistance of about $8.9 \Omega$. At the current bias $1.7 \mu \mathrm{A}$ of the minimum differential resistance, the voltage drop along this $180 \mathrm{~nm}$ coherence length is $15 \mu V$. This latter value is close to the Thouless energy derived from Fig. 2.

If we want to carry out a thorough quantitative analysis of our results, we encounter several difficulties : (i) A part of the resistance drop below the $\mathrm{Al}$ superconducting transition should originate from the local short-circuit by the $\mathrm{Al}$ island. A complete description would require taking into account the current redistribution in the Co thickness beneath the $\mathrm{Al}$ wire. (ii) The amplitude of the resistance drop is relatively small in comparison with the expected $15 \%$ variation for the resistance of the regions affected by the proximity effect. This could be related to the fact that we do not have good reservoirs injecting at a given energy from a well-defined distance, but diffusively distributed phase breaking and inelastic events along the $\mathrm{F}$ wire.

Let us discuss the possible origin of the excess resistance, above the normal state residual resistance, at low temperature. This result is in agreement with the theoretical prediction of Stoof and Nazarov, that the zerotemperature and zero-voltage resistance of a N-S structure should exceed the normal-state resistance if repulsive e-e interactions are present. Following this viewpoint, our experiment would reflect a direct influence of the e-e interaction on a metallic resistance. From our data, we could extract a value for the electron-electron interaction parameter. Another quite different explanation for the excess resistance at low temperature could be the screening of magnetic field by the superconductor in contact with the ferromagnet. The modification of the magnetic domain configuration near the F-S interface 19 could enhance the resistivity in this region.

In conclusion, we have observed a proximity effect on the dissipative transport in a ferromagnetic metal in contact with a superconductor. Our results are in agreement with the early works of Petrashov 12 and LawrenceGiordano 13 . From this work, we can assert than the behaviour described in Ref. 13 is due to a superconducting proximity effect in the ferromagnetic metal. The energy dependence of the effect has been probed through the temperature and voltage dependence of the resistance. The decay length for the coherence effect appears to be about $180 \mathrm{~nm}$ in the Co film. This value is of the order of the expected size of the magnetic domains in such films. The excess resistance, above the normal-state value, at zero voltage and temperature, could be explained by interaction effects.

We thank B. Spivak, P. Butaud and J. Caulet for stimulating discussions, D. Lafont, D. Mariolle (CEA-LETI) and Th. Fournier for the SEM micrograph of the samples, and J. Gilchrist for proof-reading the manuscript.

${ }^{1}$ P. G. de Gennes, Superconductivity of metals and alloys (Benjamin; N. Y.), 1964.

${ }^{2}$ V.T. Petrashov, V.N. Antonov, P. Delsing, and T. Claeson, Phys. Rev. Lett. 70, 347 (1993); Phys. Rev. Lett. 74, 5268 (1995).

${ }^{3}$ A. Dimoulas, J.P. Heida, B.J. van Wees, T.M. Klapwijk, W.v.d. Graaf, and G. Borghs, Phys. Rev. Lett. 74, 602 (1995).

${ }^{4}$ H. Courtois, Ph. Gandit, D. Mailly, and B. Pannetier, Phys. Rev. Lett. 76, 130 (1996).

${ }^{5}$ P. Charlat, H. Courtois, Ph. Gandit, D. Mailly, A. Volkov, and B. Pannetier, Phys. Rev. Lett. 77, 4950 (1996); Czech. J. of Phys. 46 S6, 3107 (1996)

${ }^{6}$ S.N. Artemenko, A.F. Volkov, A.V. Zaitsev, Sol. St. Comm. 30, 771 (1979).

7 T. H. Stoof and Y. Nazarov, Phys. Rev. Lett. 76, 823 (1996).

${ }^{8}$ A. A. Golubov, F. K. Wilhelm, and A. D. Zaikin, Phys. Rev. B 55, 1123 (1997).

${ }^{9}$ F. Zhou, B. Spivak, and A. Zyuzin, Phys. Rev. B 52, 4467 (1995).

${ }^{10}$ R. F. Hoyt and A.C. Mota, Sol. St. Comm. 18, 139 (1976).

${ }^{11}$ B. Spivak and A. Zyuzin, preprint condmat 9602005.

${ }^{12}$ V. T. Petrashov, JETP Lett. 59, 551 (1994).

${ }^{13}$ M. D. Lawrence and N. Giordano, J. Phys. Condens. Matter 8, 563 (1996)

${ }^{14}$ F. K Wilhelm, A. D. Zaikin and H. Courtois, Phys. Rev. Lett. 80, 4289 (1998).

${ }^{15}$ W. Wernsdorfer, K. Hasselbach, D. Mailly, B. Barbara, A. Benoit, L. Thomas and G. Suran, J. Mag. Mag. Mat. 145, 33 (1995)

${ }^{16}$ G. Tatara, H. Fukuyama, Phys. Rev. Lett. 78, 3773 (1997)

${ }^{17}$ E. A. Demler, G. B. Arnold and M. R. Beasley, Phys. Rev. $B$ 55, 15174 (1997).

${ }^{18}$ M. J. M. de Jong and C. W. J. Beenakker, Phys. Rev. Lett. 74, 1657 (1995).

19 A. I. Buzdin and L. N. Bulaevski, Sov. Phys.JETP 67, $576(1988)$ 